SHS Web of Conferences 21, 01001 (2015)

DOI: $10.1051 /$ shsconf/ 20152101001

(C) Owned by the authors, published by EDP Sciences, 2015

\title{
« Vie » et « vivant » : perspectives épistémologiques
}

\author{
Michèle dell'Angelo-Sauvage ${ }^{1}$ \\ UMR STEF ENS Cachan, Université Paris Est Créteil, ESPE 36 rue Charpak, F77587 Lieusaint
}

\begin{abstract}
Résumé. Des idées des philosophes de la Grèce ancienne, à celles que suggèrent les avancées contemporaines de la biologie, différentes conceptions de la vie et du vivant sont proposées sans qu'un consensus ne soit obtenu. Différents auteurs ont présenté la vie comme l'affaire des philosophes, sous ses aspects politiques et sociaux, tandis que le vivant était l'affaire des biologistes préoccupés du fonctionnement des organismes (Canguilhem, 1990; Fassin, 2000; Jacob, 1970; Pichot, 2011). Mais cette distinction est actuellement rediscutée (Cherlonneix, 2013; Morange, 2013). D'autres auteurs mettent en contraste les approches réductionnistes et holistes et présentent les conséquences de ces points de vue. Dès 2006, Guespin-Michel et Stewart envisageaient un changement de paradigme inévitable face aux modifications de méthodes dans différents groupes de recherche. Nous proposons de porter un regard croisé sur les concepts de vie et de vivant dans le but d'éclaircir les fondements épistémologiques sous-jacents à un enseignement relatif au vivant : observe-t-on une influence réciproque des conceptions de «vie » et de «vivant» dans les différents courants de pensée ? Les changements épistémologiques liés aux observations faites à différentes échelles biologiques modifient-ils les réflexions philosophiques, scientifiques et sociétales? Qu'en est-il actuellement de la distinction vie/vivant ?
\end{abstract}

\begin{abstract}
Life' and 'living': epistemological perspectives. From the ideas of the philosophers of Ancient Greece to the questions raised by contemporary advances in biology, different conceptions of life and living have been put forward but no consensus can be reached. Different authors have presented life, in its political and social aspects, as a subject matter for philosophers whereas living was a subject matter for biologists whose concern is the functioning of living organisms (Canguilhem, 1990; Fassin, 2000; Jacob 1970 Pichot, 2011). But this distinction is being discussed again (Cherlonneix, 2013; Morange, 2013). Other authors contrast reductionist and holistic approaches and present the consequences of these views. In 2006, GuespinMichel and Stewart considered an inevitable paradigm shift, in response to the changes in methods in different research groups. We propose to bring a fresh perspective to the concepts of life and living in order to clarify the epistemological foundations underlying a teaching concerning life: is there a reciprocal influence of the conceptions of «life» and «living » in the different schools of thought? Can the epistemological changes linked to the observations made at different biological scales alter philosophical, scientific and societal reflections? What does the distinction between life and living now consist in?
\end{abstract}

\footnotetext{
${ }^{1}$ Auteure de correspondance : michele.dellangelo@u-pec.fr
} 


\section{Introduction}

La question de la vie et du vivant a souvent été discutée sans recevoir de réponse faisant consensus. Par-delà la succession de définitions que nous rappellerons rapidement et sans volonté d'exhaustivité, c'est toute une conception de la vie et du vivant qui est questionnée. Avec un regard de biologiste, que peut-on dire des approches historiques de philosophes, sociologues, anthropologues ? Quelles conceptions de la vie et du vivant sous-tendent-elles?

Nous assistons actuellement à d'importants changements épistémologiques liés aux observations faites à différentes échelles, avec de nouveaux outils ${ }^{2}$, de nouveaux questionnements sociétaux et politiques. Peuvent-ils annoncer un véritable changement de paradigme comme l'envisagent GuespinMichel et Stewart dès 2006 [1] ? Dans l'introduction de son livre Cherlonneix (2013) [2] annonce L'hypothèse de travail interrogée - et nous ne sommes pas tous d'accord sur les réponses...- est qu'un renversement de paradigme concernant les représentations du vivant est à l'œuvre en biologie. (p. xii). Ce sont certains de ces désaccords que je propose de présenter ici, partant de l'idée que l'ancienne partition entre la vie qui serait l'affaire des philosophes, sous ses aspects politiques et sociaux, tandis que le vivant serait celle des biologistes préoccupés du fonctionnement des organismes (Canguilhem, 1990 [3]; Fassin, 2000[4]; Jacob, 1970 [5] ; Pichot, 2011 [6]) n'est plus tenable aujourd'hui. Une simple question comme : l'embryon est-il en vie dès sa conception ? (Wolfe, 2013) [7] repose sur des études biologiques, mais renvoie à des aspects philosophiques. Et c'est l'ensemble de ces réflexions qui conditionnent des positionnements éthiques rendant par exemple possible les expériences scientifiques sur les cellules totipotentes, le diagnostic préimplantatoire ou les soins utilisant des cellules souches. Un autre exemple est fourni par Ameisen (2013) [8], qui aborde la question de la vie par celle de la mort. Il s'interroge sur le sens qu'elle prend lorsqu'il s'agit de reproduction asexuée, pour les végétaux, les tout petits animaux, alors qu'il n'y a aucune différence génétique entre les descendants et les parents. Si le vivant est nature, et la nature, natura, littéralement 'ce qui est en train de naître' cela fait trois à quatre milliards d'années que le vivant est en train de naître et de se métamorphoser... La vie en tant que telle n'est jamais morte (p. 4). La définition de la vie à l'échelle de l'organisme est-elle la même qu'à l'échelle d'une cellule où d'une population?

Une inquiétude provient de l'indifférence de certains biologistes qui refusent de prendre part au débat éthique et se retranchent derrière un rôle de technicien du progrès scientifique. Que de fois n'aije entendu lors de débats publics des scientifiques, des biologistes, pressés d'aborder la question de la nature de la vie, répondre que cette question était une question philosophique et céder la parole aux philosophes (p. 41) nous dit Morange (2013) [9]. Tournier (2005) [10] pose le problème du côté du pouvoir ainsi laissé à la technique et parle d'une dérive dangereuse à relier à la parcellisation des savoirs qui, de plus en plus pointus, demeurent aussi beaucoup plus restreints (p. 13).

Cet article propose donc de s'appuyer sur des approches épistémologiques de différentes disciplines pour chercher à comprendre ce que certaines avancées scientifiques tendent à modifier dans la perception de la vie et du vivant.

\section{Une construction historique des concepts de vie et de vivant}

Les concepts de vie et de vivant ont été utilisés et définis depuis les philosophes de la Grèce antique. Les changements intervenus dans la compréhension des structures et des fonctionnements du vivant ont conduit les chercheurs à adopter parfois des positions très différentes plus ou moins en opposition. Ainsi, on ne peut pas retracer une histoire au sens de modifications successives avec un début et une fin, mais plutôt au sens de réflexions centrées sur des aspects, des intérêts, et parfois même des ${ }^{2}$ Se décrivant par une multitude de vocables: Autopoïèse, biologie intégrative, biologie des systèmes,
épigénomique, post génomique, entre autres. 
croyances, différents. Des définitions, reprises ci-dessous, ont particulièrement marqué les pensées humaines.

\subsection{Des tentatives de définition}

Très tôt, Aristote ${ }^{3}$ définit la vie comme un processus temporel avec un début et une fin: nous entendons par vie le fait de se nourrir, de crôttre et de dépérir par soi-même [11]. C'est ce que l'on nomme la conception animiste, présente chez le jeune enfant, mais aussi nous le verrons dans des positionnements contemporains. Il faut attendre le $17^{\text {ème }}$ siècle pour rencontrer une définition qui perdure aussi sous certaines formes, celle de Descartes. Il définit le vivant par les caractéristiques de la vie en écrivant l'être vivant est une machine complexe, faite d'organes assurant les fonctions vitales : nutrition (respiration, alimentation, croissance, renouvellement), reproduction et défense [11]. C'est la conception mécaniste. Au $18^{\text {ème }}$ siècle Bichat ${ }^{4}$ définit la vie par un conflit entre un corps composé de tissus de structure et de propriétés spécifiques (élasticité, contractilité, sensibilité) et un environnement. La vie est l'ensemble des fonctions qui résistent à la mort [11]. On parle de conception vitaliste. Très rapidement ensuite, à la charnière du $18^{\text {ème }}$ et du $19^{\text {ème }}$ siècle, Lamarck rattache le vivant à la présence de matière que l'on qualifie aujourd'hui d'organique. On parle encore actuellement de cette conception dite matérialiste. Aucune de ces définitions ne peut être retenue seule, mais chacune aborde certaines caractéristiques, parfois de la vie, parfois du vivant. Un des éléments de comparaison partagés est l'organisation, à différentes échelles, avec différents prismes d'observation suivant les champs disciplinaires.

\subsection{Des approches par l'organisation}

Si l'on interroge les scientifiques, les philosophes, les éthologues, différentes façons de concevoir des visions hiérarchiques du vivant apparaissent. Même le mot animalité, Chevrier et Maurice (2004) [12] nous disent que ce sont les philosophes qui l'ont créé pour définir l'Homme. Il correspond au moment où le mot "homme " ne peut plus être appliqué à la réalité de l'homme. C'est une marque d'infamie à la surface de l'espèce humaine (p. 12).

Plusieurs visions perdurent actuellement qui s'affrontent et discutent la question des droits de l'animal (les plantes sont très peu évoquées). Nous allons parcourir les positions de plusieurs champs disciplinaires en reprenant l'historique de leur construction pour mieux en comprendre l’épistémologie.

\subsubsection{Des conceptions souvent hiérarchiques de la vie et du vivant pour les philosophes}

Les philosophes s'interrogent sur les relations entre l'Homme et les autres animaux. Très tôt ils introduisent une forme de hiérarchie du vivant en discutant de l'âme. Une âme d'origine divine chez Platon, qui peut passer de l'animal à l'Homme; des âmes qui permettent de considérer l'Homme comme supérieur pour Aristote :

- les végétaux ont une âme végétative. Ils se reproduisent et se nourrissent.

- les bêtes ont une âme sensitive. Elles ont en plus des sensations, de l'imagination, de la mémoire, une intelligence rudimentaire.

- les Hommes ont une âme intellective. Ils ont en plus la raison, d'où leur position au sommet des espèces (Baratay, 2003 [13]; Canguilhem, 1990 [11]).

Pendant longtemps les mauvais traitements aux animaux trouvent donc leur justification dans le fait qu'ils sont irraisonnables et instinctifs. Au $17^{\text {ème }}, 18^{\text {ème }}$ siècle, la discussion se déplace vers la

\footnotetext{
${ }^{3}(-384-322)$

${ }^{4}$ Recherches physiologiques sur la vie et la mort (1800).
} 
souffrance de l'animal, avec Descartes qui décrit les bêtes comme des sortes de machines insensibles à la douleur, puis avec Rousseau (1755) [14], qui distingue l'Homme par la pitié : la répugnance naturelle à voir souffrir tout être sensible, et précise-t-il : cette commisération sera d'autant plus énergique que l'animal spectateur s'identifiera avec l'animal souffrant (p. 36). Ici, c'est donc le droit de l'Homme qui est limité par la souffrance de l'animal. La distinction par la souffrance est concrétisée en 1978 par l'élaboration de la déclaration universelle des droits de l'animal à l'UNESCO qui distingue en particulier les vertébrés des autres animaux. Le débat se poursuit en particulier dans le champ de l'expérimentation animale.

La plupart des philosophes persistent à considérer l'Homme comme supérieur aux autres espèces et l'argumentation s'oriente vers la capacité à transmettre une culture. En 1992, Ferry [15] précise : Si nous ne possédions pas la faculté de nous arracher à cette culture traditionnelle qui s'impose à nous comme une seconde nature, nous resterions, tout comme les bêtes, régis par des codes naturels (p. 47). Cet élément déterminant est aussi repris par des linguistes et les anthropologues.

Néanmoins de nouveaux courants de pensée apparaissent avec les utilitaristes. Peter Singer par exemple considère que dès qu'un être a la capacité à éprouver du plaisir et de la peine, une considération morale lui est due (Larrère, 2004 [16]; Goffy, 2007 [17]; Singer, 2007 [18]). Il condamne ainsi l'élevage d'animaux pour la nourriture, l'expérimentation animale, la chasse, le commerce d'animaux sauvages et remet en cause le caractère sacré de la vie humaine : il faut protéger les animaux supérieurs en tant qu'espèces biologiques, mais on peut tuer des individus biologiques dans certains cas : avortement, euthanasie de nouveaux nés mal formés ou « légumes ».

D’une façon générale, l'Homme persiste à être considéré comme une espèce «à part» par les philosophes, et le plus souvent comme supérieur avec des arguments qui ont changé parallèlement aux découvertes en biologie. Nous allons voir qu'actuellement d'autres arguments conduisent à un résultat similaire en lien avec des intérêts économiques.

\subsubsection{Les approches juridiques, de nouvelles hiérarchies}

On constate une nouvelle façon de considérer le vivant en introduisant des notions comme le profit ou la gloire personnelle. C'est alors en termes juridiques que l'on envisage de créer de nouvelles hiérarchies des espèces. On distingue l'animal domestique, considéré comme un «meuble» dans le Code civil français jusqu'en 2014, et l'animal sauvage qui peut être espèce protégée, "gibier » ou «nuisible». La situation se complique encore, lorsque l'on tente de classer les produits et les éléments du corps humain, les inventions biotechnologiques, l'embryon humain. Depuis 1980, date à laquelle les États-Unis obtiennent la brevetabilité de micro-organismes ${ }^{5}$, différents vivants plus ou moins modifiés ont pu être brevetés (Hermitte, 1990 [19]; Lecourt, 2003 [20]). Récemment l'Office européen des brevets (OEB) vient d'octroyer plusieurs brevets pour des légumes sélectionnés, mais non modifiés génétiquement au profit de firmes internationales.

Envisager le droit de breveter des êtres vivants, définir des règlementations à propos d'organes ou d'embryons, dénote une grande évolution du rapport aux vivants qui ne sont plus seulement envisagés en tant qu'êtres, mais aussi en tant qu'éléments fonctionnels du vivant (organe, source de cellules totipotentes...), ou même en tant que source de gloire personnelle et de revenus.

Il apparaît aussi une différenciation très nette entre les vivants : ceux qu'il semble urgent de doter de droits pour les protéger, ce qui dénote une importance socialement reconnue; ceux dont l'individualité n'apparaît pas et qui sont seulement considérés en tant qu'espèce ; les autres enfin, les plus nombreux, qui ne font l'objet d'aucune loi, d'aucune reconnaissance bien qu'ils soient indispensables dans les écosystèmes.

\footnotetext{
5 Il s'agissait d'une bactérie manipulée par Amanda Chakrabarty pour consommer des hydrocarbures. L'accord avait été donné parce que la bactérie n'existait pas comme telle dans la nature.
} 
La « vie » et le « vivant » : de nouveaux défis à relever dans l'éducation

Cette différenciation ressort aussi des études des biologistes, éthologues, ethnologues et sociologues.

\subsubsection{Les approches des éthologues, des ethnologues et des anthropologues}

L'argument de la possession ou non de la raison, la conscience de soi, a souvent été employé pour distinguer l'Homme des autres vivants. Darwin (1872) [21] reconnaît des facultés mentales et le sens moral des animaux (p. 270). Une analyse plus détaillée est fournie par le philosophe et éthologue Lestel (2004, p. 85) [22]. Il distingue les animaux dont les comportements sont en partie déterminés par des raisons et non par des causes, et qui peuvent transformer leurs préférences de façon cohérentes dans le temps. Il établit ainsi toute une distinction entre des sujets :

- faibles autonomes: qui n'ont pas besoin de l'Homme pour qu'émerge leur individualité (chimpanzés)

- faibles hétéronomes : qui ont besoin de l'Homme (oies)

- hétéronome fort : qui sont des personnes (singes parlant)

Un autre attribut discriminatoire est l'instinct qui s'oppose à la culture. Cuvier disait que l'instinct et l'intelligence varient en raison inverse l'un de l'autre (p. 69) [21].

Deux courants s'opposent encore (Baratay, 2003) [13]:

- celui de la psychologie cognitive qui reprend des idées très proches de celles de l'école behavioriste aux USA en 1930 : Les organismes, dont l'homme, sont pensés comme des machines neuronales confrontées à de l'information à traiter. Leur connaissance vient de leur capacité à combiner des représentations pour faire des opérations logiques (p. 182).

- celui de l'éthologie qui se développe au début du $20^{\mathrm{eme}}$ avec, entre autres, Lorenz et ses études sur l'attachement du jeune, le phénomène de l'empreinte.

En 1953, Satsuo Mito montre l'existence et la transmission d'une culture chez les macaques. (Vandeginste, 2001) [23]

Certains éthologues vont plus loin en attribuant aux animaux des sentiments complexes comme la jalousie; des émotions intellectuelles telle la curiosité; une aptitude à la vie sociale, aux raisonnements, à la conscience de soi, au progrès, à la notion du beau. De Waal (1992) [24] montre par exemple que les grands singes utilisent des plantes médicinales. Ils font des efforts pour se rabibocher après une dispute, et savent nouer des alliances pour se rendre populaires et accéder au pouvoir. Il observe et expérimente des comportements de partage et d'entraide, de compassion même. Les chimpanzés cherchent à apaiser des conflits et parfois défendent les plus faibles (De Waal, 1992b) [25]. Ces comportements relèvent d'une morale. En 1997 [26] De Waal risque même l'hypothèse selon laquelle notre morale actuelle pourrait s'être construite à partir de bases préexistantes chez les ancêtres que nous avions en commun avec les chimpanzés il y a dix millions d'années. Cela s'oppose radicalement à l'idée commune d'une nature mauvaise, animale que notre culture nous aide à vaincre. Une autre approche vient par exemple du philosophe Merleau-Ponty (1998) [27] qui montre que l'existence d'un dialogue entre les animaux au moment des parades sexuelles permet de parler d'une culture animale. Le langage a beaucoup été étudié chez les singes et les dauphins. Celui des oiseaux montre actuellement une transmission par les parents et des motivations qui dépassent le fonctionnel.

Plus étonnant les éthologues considèrent que les herbivores sont des prédateurs et qu'ils détruisent des populations (la vache faisant disparaitre l'herbe dont elle arrache la racine). Les végétaux sont ainsi mis sur le même plan que les animaux. Des espèces sont requalifiées, comme les champignons distingués des végétaux (les végétariens doivent-ils encore les consommer ?).

Il semble donc bien que pour certaines espèces il y ait véritablement une culture qui s'élabore et se transmet. Un par un les arguments en faveur d'une primauté de l'Homme sur les autres êtres vivants 
tombent. Bien sûr, cela remet en question toute la façon de concevoir l'Homme par rapport aux autres animaux. Cela modifie aussi le rapport au vivant construit à l'école.

\subsubsection{Les approches en référence à l'origine de la vie et à l'évolution}

Les scientifiques ont présenté différentes «visions » de l'évolution dont on retrouve une persistance dans les représentations des jeunes élèves et certains propos d'adultes. La plus ancienne (Lecointre et

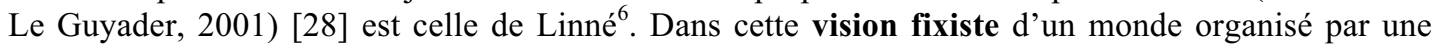
volonté divine, l'Homme est représenté tout en haut, et en dessous, de plus en plus bas, sont disposées les espèces qui ont de moins en moins de points communs avec lui. C'est bien souvent cette vision que l'on retrouve dans les représentations des jeunes enfants et elle perdure aussi de façon insidieuse lorsque l'Homme est dit «plus évolué » que les autres espèces. Avec Lamarck ${ }^{7}$ s'impose une vision transformiste de la classification du vivant. Elle repose sur la transmission des caractères acquis. Bien que totalement niée par les découvertes en biologie moléculaire, en génétique et même en embryologie, cette vision perdure de façon masquée dans beaucoup de conceptions d'élèves et d'adultes. Elle peut être involontairement induite par des enseignements de SVT (voir le cas de la phalène du bouleau (2009) [29] et l'étude de Kuster et Lange (2009) [30]). La vision évolutionniste de Darwin repose sur la transmission des caractères anciens et les innovations évolutives sélectionnées par le milieu. Dans un premier temps elle a conduit à la représentation des relations entre les vivants en terme de généalogie (parents - enfants). Des idées comme «nous descendons tous des poissons » ou "l'homme descend du singe » ont été vulgarisées et sont maintenant très tôt redites aux enfants par les parents, les médias. Beaucoup de livres encore utilisés en reproduisent les classifications, avec par exemple d'un côté « les vertébrés », bien détaillés et prenant la plus grande place, et de l'autre, « les invertébrés » (les plus nombreux sur la Terre), sur un tout petit espace.

Actuellement ce sont des relations en termes de phylogénies qui sont représentées. Les caractères communs partagés sont relevés. On définit des liens de parenté entre des espèces ayant eu un " ancêtre commun ». Ils sont en quelque sorte cousins. Ces nouvelles conceptions de la classification du vivant ont été introduites dans les programmes français de l'école primaire et du collège. Elles constituent un bouleversement assez important des habitudes (les poissons, par exemple n'apparaissent plus en tant que tels), mais constituent une avancée importante pour placer l'Homme parmi les autres vivants. On peut donc considérer que cette relation hiérarchique a été « historiquement créée » et " biologiquement détruite». Mais combien de temps faudra-t-il pour que ces conceptions quittent l'école et les milieux spécialisés pour arriver dans le « grand public »?

La façon d'envisager l'évolution donne aux vivants une importance relative différente. Encore en 2003, Morange [31] nous rappelle que certains biologistes considèrent «la vie comme déroulement d'un programme naturel de complexification (p. 32). Gould (2001) [32] précise que peu de personnes sont prêtes à admettre que l'évolution ne devait pas nécessairement conduire à nous. C'est cette remise en question de la place de l'homme comme sommet prédictible de l'évolution qui est le message le plus perturbant de la révolution darwinienne (p. 24). Il étudie en 1997 [33] « l'explosion cambrienne » qui a vu surgir toutes les espèces pluricellulaires il y a environ 600 millions d'années, et montre qu'un petit nombre de lignages, dont celui de l'Homme, se sont complexifiés, mais que c'est une tendance mineure. Morange (2003) reprend pour sa part l'explication de certains biologistes qui remarquent que ce sont les formes vivantes actuelles les plus simples qui sont souvent les plus anciennes et se reproduisent le plus rapidement, de sorte qu'elles ont eu «le plus de possibilités de s'éloigner de leur état initial (p. 32) [31]. La réponse des biologistes n'est donc pas unitaire et de nature à clore les débats, mais elle suggère l'idée d'une différence de statut entre des vivants plus ou moins « aboutis ».

\footnotetext{
${ }^{6}$ Carl von Linné, 1707 - 1778.

${ }^{7}$ Lamarck, $1744-1829$.
} 


\subsubsection{Les approches en référence au concept d'organisme}

Le concept d'organisme commence à être utilisé au $17^{\text {ème }}$ siècle, mais il sous-tend suivant les auteurs des compréhensions du vivant parfois diamétralement opposées. Ainsi Wolfe (2013) [7] nous rapporte que Leibniz y voit un synonyme de ce qu'il nomme machine de la nature, alors que pour Stahl il s'agit de produire un modèle du vivant non mécanique, un tout organique à l'individualité intrinsèque (p. 270). D’une façon générale, il distingue trois approches possibles: celles qui s'opposent au réductionnisme, celles qui visent à modéliser le vivant ou celles qui veulent rendre compte de notre incapacité constitutive à saisir une " essence » ou " origine " de la vie et de tout être vivant (p. 274). Il les nomme : mécanisme, organicisme et instrumentalisme. Les mécanistes veulent définir l'organisme au travers de «lois», de régularités systémiques. Ils sont rejoints par les organicistes ${ }^{8}$, mais ceux-ci considèrent que les modèles mécaniques ne peuvent pas rendre compte du vivant. Ils s'accordent pour définir l'organisme plutôt comme une forme d'intelligibilité, une manière de voir et rendre intelligible certaines parties de la nature (p. 274). Les instrumentalistes considèrent que les seules choses réelles sont les entités appartenant à la physique fondamentale.

L'opposition que l'on rencontre le plus souvent cependant est celle entre une conception mécaniste dite aussi réductionniste, qui cherche à rendre compte des fonctions par les seules structures, et une conception vitaliste dite aussi globaliste qui affirme que l'organisme ne se réduit pas à ses constituants (Simard, Harvey et Samson dans cet ouvrage) [34]. Nous verrons qu'actuellement elles s'affrontent, mais aussi se croisent.

Nous retrouverons ces approches au travers des avancées scientifiques remettant actuellement les compréhensions de la vie et du vivant en cause.

\section{Vie / vivant: des remises en question actuelles}

Pour argumenter un changement de paradigme, Ameisen (2013) [8] revient sur la notion d'anomalie proposée par l'historien des sciences Kuhn [35], comme des observations que les concepts scientifiques de l'époque ne permettent pas d'expliquer. Il utilise la mort cellulaire massive intervenant parfois au cours du développement embryonnaire, connue depuis un moment, mais jamais vraiment étudiée, tant l'idée qu'une disparition s'opposait à celle de la construction de notre corps. $\mathrm{Ce}$ n'est souvent pas la découverte d'anomalies qui provoque un changement de paradigme, mais un changement de paradigme qui permet de reconnaitre, rétrospectivement, l'existence d'anomalies qu'on refusait jusque-là, de prendre en compte ([8] p. 9).

Nous allons envisager les avancées scientifiques actuelles sous l'angle des anomalies pointées et des nouvelles questions posées, notamment au regard de la génétique, de la biologie intégrative, de l'embryologie, de l'évolution et de l'immunologie.

\subsection{En relation avec les avancées de la génétique}

Après un passage de ce que Morange (2003 [31], 2013 [9]) et Atlan (2003) [36] nomment le tout cellulaire, au tout moléculaire puis au tout génétique, les résultats du séquençage du génome humain ont montré que la connaissance de l'ADN ne suffisait pas pour expliquer le développement et le fonctionnement des organismes.

Le questionnement qui s'est alors développé porte sur l'environnement. On a ainsi montré qu'avec le même génome, mais dans deux environnements différents, les phénotypes pouvaient être opposés. Le cas des œufs de tortue qui peuvent donner un mâle ou une femelle en fonction de la température

\footnotetext{
${ }^{8}$ L'embryologiste Hans Driesch (1867-1941); Kant (1724-1804); le neurologue Kurt Goldstein (1878-1965), et le philosophe contemporain Daniel Dennett (1942-)
} 
est souvent cité pour l'illustrer. Les gènes changent d'activité en fonction de facteurs intra cellulaire et extracellulaires. Ces études portent le nom d'épigénétiques. Pour expliquer ces phénomènes, Atlan (1999) [37, p. 37] décrit un réseau évolutif dont la structure change au fur et à mesure de son fonctionnement : les gènes modifient le milieu cellulaire, donc le fonctionnement de son propre réseau biochimique qui, en retour, modifie l'état d'activité du gène. La vie n'est plus pré définie. Elle n'est plus non plus individuelle puisqu'elle dépend des signaux reçus des autres cellules.

Le gène reste un élément fondamental pour décrire la cellule, mais sa connaissance ne suffit plus pour envisager son devenir. Élément d'un réseau elle communique avec les autres cellules.

Mais ces découvertes qui marquent l'entrée dans une modalité de questionnement propre à ce que l'on nomme la post génomique, ne peuvent être dissociées des technologies qui ont permis de les étudier et de la bio-informatique qui permet de traiter l'abondance des résultats obtenus.

\subsection{En relation avec les avancées en biologie intégrative}

C'est ainsi en relation avec de nouveaux besoins que s'est développée la biologie intégrative. Ce courant de recherche scientifique croise les regards des mathématiques, de la physique et de la biologie. Elle tend à prendre en compte les différents niveaux d'organisation et leurs interactions. Depuis les années 80 avec Varela ${ }^{9}$ et Maturana ${ }^{10}$ la vie est questionnée en tant que système complexe de réactions physico-chimiques se déroulant dans la cellule eucaryote. Ces auteurs parlent de système autopoḯtique c'est-à-dire capable de se produire lui-même, en permanence en interaction avec son environnement grâce à un flux externe de molécules et d'énergie.

En s'appuyant sur la théorie des systèmes complexes (Kitano, 2002 [38]; Liu, 2005 [39]), il s'agit de décrire et expliciter le fonctionnement du vivant dans l'espace moléculaire, cellulaire, tissulaire, etc., et aussi les interactions entre les facteurs environnementaux et l'organisme (Coquidé, dell'Angelo, Dorey, Fortin, Gallezot, Henocq, Kalali, Lange, Rumelhard, 2012) [40]. Posant des questions sous des angles statistiques, chimiques et biochimiques, mécaniques, énergétiques, communicationnels, ces études pointent des interactions possibles ou incontournables. Les approches déterministes sont englobées dans les approches probabilistes (décrites comme les situations à probabilité proche de 1, comme le suggère Heams (2013, p. 144) [41].

Dans certains cas elles peuvent réintroduire des conceptions réductionnistes de la vie comme celles du biophysicien Chauvet ${ }^{11}$ pour lequel les systèmes biologiques sont des systèmes physiques pourvus de propriétés complémentaires, liées à l'existence d'interactions fonctionnelles. Il écrivait sur son site: La vie d'un organisme vivant se résume à un ensemble d'interactions de multiples mécanismes élémentaires qui se déroulent au bon moment, au bon endroit [42].

Mais elles conduisent le plus souvent à des conceptions stochastiques telles que le réseau évolutif d'Atlan dont nous avons parlé. Sur un exemple simple, Coquidé et al. (2012) [40] décrivent le passage d'une approche réductionniste par le modèle analogique clef-serrure, conçu comme une complémentarité " active " permettant l'emboîtement topologique de deux molécules dans l'espace, à une approche incluant des phénomènes dynamiques d'interactions moléculaires. S'adaptant l'une à l'autre les molécules changent leurs conformations 3D et ainsi les modalités et l'ampleur de la réponse. Ce qui semble être juste l'évolution d'un modèle constitue une sorte de révolution dans la façon de considérer le vivant. Dans la première situation que Kupiec et Sonigo (2000, p.71) [43] nomment le paradigme réductionniste de la biologie moléculaire, les gènes conduisent à la synthèse de protéines qui interagissent pour former les constituants de la cellule, laquelle forme avec d'autres les tissus puis les organes et enfin l'organisme entier. Dans la deuxième situation, nommée souvent

\footnotetext{
${ }^{9}$ Francisco Varela, 1946 - 2001.

${ }^{10}$ Humberto Maturana, $1928-$.

${ }^{11}$ Gilbert A. Chauvet, $1942-2007$.
} 
environnementaliste, des données chimiques telles que la vitesse d'association des molécules ou la stabilité d'un complexe interviennent. L'ADN peut alors conduire à plusieurs structures moléculaires qui créent un environnement susceptible de moduler l'expression des gènes aussi bien que les éléments macroscopiques. Une question nouvelle est alors posée: comment en utilisant des mécanismes aléatoires, des tissus, des organismes semblables d'une génération à l'autre peuvent-ils se constituer?

\subsection{En relation avec les avancées de l'embryologie}

Ce qui a longtemps prévalu dans l'explication de l'ontogenèse est l'interaction entre les cellules, l'une émettant un signal (l'inducteur) l'autre réagissant. Cette approche a été complétée par la théorie des gradients morphogénétiques, qui s'appuie sur l'idée que la concentration locale de substances morphogènes induirait des différenciations cellulaires particulières (Kupiec et Sonigo, 2000, p. 90.) [43]. Dans les années 1960, le travail de Jacob et Monod sur Escherichia coli déplace la question à l'échelle des gènes. La découverte de ceux codant pour la fabrication des trois enzymes lui permettant d'utiliser le lactose, conduisent à des modèles impliquant des gènes régulateurs ${ }^{12}$. Il est alors possible de penser la différenciation embryonnaire à l'échelle moléculaire. Avec les gènes homéotiques s'installe l'idée que des gènes sont responsables de la localisation des organes au cours du développement. Des drosophiles mutantes développent sur un segment, des organes normalement présents sur d'autres segments du corps, par exemple des pattes à la place des antennes ${ }^{13}$. Parallèlement les études épigénétiques questionnent les mécanismes ontogénétiques. En 1988, le lien est fait avec la production d'une protéine suivant un gradient depuis la partie antérieure jusqu'à la partie postérieure, par le jeu de 2 gènes antagonistes. Dupont (2013) [44] parle d'une double régulation à la fois génétique et épigénétique: Toute une variété de phénomènes dépendant de l'environnement modulent l'expression des gènes au cours du développement (phénomène d'empreinte, effet maternel, effet de position, etc.) (p. 264).

Là aussi différents domaines s'unissent pour questionner autrement le vivant: la génétique, l'embryologie et l'évolution, mais aussi l'anatomie et la paléontologie. C'est ce que l'on nomme évodévo, la génétique évolutive du développement.

\subsection{En relation avec les conceptions de l'évolution}

L'évolution des espèces est de façon assez consensuelle expliquée par des phénomènes génétiques (mutation, recombinaison, duplication...) conduisant à une diversification du vivant en lien avec une sélection du milieu environnant telle que Darwin l'a introduite.

Certaines observations telles que nous les décrit Fortin dans cet ouvrage [45], montrent de nouveaux mécanismes de la diversification du vivant.

À l'échelle intra cellulaire, l'idée d'une compétition entre des molécules issues de la variabilité aléatoire de l'expression des gènes, suivie d'une sélection de certaines en fonction de leur nombre ou de leur compatibilité s'accorde bien avec une conception darwinienne de l'évolution (Kupiec, 2009) [46].

La question se pose alors différemment, et rejoint celle que nous avons vue dans les cellules. Il s'agit non plus de comprendre comment les cellules se différencient, mais comment elles peuvent se stabiliser pour former un tissu par exemple. C'est ce que Kupiec nomme l'identification cellulaire, pour laquelle il fournit un modèle fonctionnel [47].

\footnotetext{
${ }^{12}$ Opéron lactose.

${ }^{13}$ Exemple: mutation de la drosophile, anténapédia étudiée au lycée.
} 


\subsection{En relation avec les avancées en immunologie}

L'immunologie a reposé très longtemps sur le paradigme très fort d'un système immunitaire propre à chaque individu (ce que l'on a appelé « le soi ») efficace pour lutter contre toute intrusion de ce que l'on a nommé « le non soi ».

Mais dans ce domaine aussi s'est posé le problème de l'influence de l'environnement. L'accent est maintenant mis sur l'immersion des individus dans un milieu avec lequel ils entretiennent des relations complexes et instables, allant de la symbiose au rejet et à la lutte à mort nous dit Moulin (2013, p. 175) [48], ce qui remet en cause le primat du sujet, c'est-à-dire l'idée que c'est l'individu qui est maître de sa défense et la déclenche lorsqu'il en a besoin.. Cette remise en question s'est faite progressivement avec la meilleure connaissance de certains mécanismes. Ainsi par exemple la phagocytose des cellules mortes revient à une attaque du soi par le soi, ce qui ne correspond pas à l'idée d'une défense, pas plus que l'activation des réponses immunorégulatrices. S'il y a des éléments microbiens qui sont considérés comme pathogènes et éliminés, il y a de plus en plus de cas connus où ils sont commensaux ${ }^{14}$. On a aussi montré les relations de symbiose avec de nombreuses bactéries au niveau de l'intestin par exemple où elles constituent un élément de défense de l'organisme parallèle et communiquant avec le système immunitaire.

Sans doute comme le dit Pradeu (2013) [49] les immunologistes préfèrent-ils jouer sur les ambiguïtés cognitives d'un système immunitaire décrit comme capable de "reconnaissance», de " discrimination » ou de " mémoire ». Le vocabulaire métaphorique, extrêmement séduisant et "vendeur» (p. 189) continu à être utilisé, mais un retournement de perspective est en route. L'organisme qui était présenté comme un tout homogène largement fermé à son environnement, doit désormais être vu comme un tout hétérogène largement construit par son environnement.

\section{Conclusion}

Les aspects plus historiques et épistémologiques de cette réflexion nous ont conduits à mettre en avant une corrélation souvent étroite entre les avancées scientifiques et les conceptions de la vie et du vivant. Que ce soit au travers des études anthropologiques, philosophiques, sociologiques ou biologiques, la place de l'Homme vis-à-vis des autres espèces est questionnée, et la considération des végétaux, phytophages, des zoophages et des omnivores est modifiée. Des différences de visées persistent cependant, qu'elles soient écologistes, éthiques, juridiques ou économistes.

Nous avons dans différents secteurs scientifiques vu apparaitre de multiples nouvelles façons de questionner la science, de questionner l'environnement, de prendre en compte l'aléatoire. Ces nouveaux questionnements sont à mettre en lien avec la découverte de nouveaux outils tant technologiques que biologiques, mais aussi avec la collaboration de différents regards, de différentes sciences. Bien souvent ils semblent marquer les limites, si ce n'est la fin d'anciens paradigmes. Pourtant deux façons de penser le vivant perdurent, une vision réductionniste dans laquelle tout ce qui existe ne serait composé que de parties, d'éléments « simples » et une vision holistique ou globaliste affirmant l'existence de systèmes sans lesquels nous ne pouvons pas comprendre les parties (Benasayag et Gouyon, 2012 [50]; Guespin-Michel et Stewart, 2006 [1]). Plus proche de la vie et tendant à l'expliquer dans sa construction et son évolution, l'opposition entre déterministes et probabilistes a tendance à s'estomper. Heams (2013) [41] la réduit au niveau d'observation auquel on se place : un foie accomplit certes sa fonction de foie, mais chaque cellule de foie a sa marge de manouvre, sa liberté biologique, en quelque sorte (p. 145). En poussant l'idée de liberté biologique à son voire Kupiec et Sonigo (2000) concluent même en disant que la vie n'est pas une machine, elle est une conjonction d'intérêts (p. 215) [43].

\footnotetext{
${ }^{14}$ Qui colonisent l'organisme sans provoquer de maladie.
} 
La vie vient comme objection au vivant et en ce sens, les questionnements philosophiques s'opposent à ceux des biologistes nous dit Rumelhard (2015) [51]. Faire de la biologie c'est mettre à l'écart la vie, prendre en compte des faits, faire des choix, mettre à l'écart ses sentiments. D'une certaine façon, la vie est un obstacle à l'activité scientifique, car bien souvent c'est elle qui «a le dernier mot »! Ainsi c'est le greffé des mains qui renonce à sa greffe, c'est le greffon venu d'un parent qui résiste mieux au rejet, c'est l'OGM qui favorise le développement de plantes parasites!

Nous resterons sur l'idée de Morange affirmant que : le vivant est histoire et que la vie ne peut être comprise indépendamment de la connaissance de cette histoire (p. 46) [9].

\section{Références}

1. J. Guespin-Michel, J. Stewart, Les théories et débats actuels en biologie : obstacles épistémologiques et obstacles économiques. In J. Guespin-Michel et A. Jacq, le vivant, entre science et marché : une démocratie à inventer (p.137 - 181) (Sylleps, Paris, 2006).

2. L. Cherlonneix, (dir.). Nouvelles représentations de la vie en biologie et philosophie du vivant. La sculpture du vivant à l'échelle de l'interdisciplinarité (De Boeck, Bruxelles, 2013).

3. G. Canguilhem. Études d'histoire et de philosophie des sciences concernant les vivants et la vie (Vrin, Paris, $19687^{\mathrm{e}}$ rééd. 1990).

4. D. Fassin. Entre politiques de la vie et politiques du vivant. Pour une anthropologie de la santé. Anthropologie et Sociétés, 24, 1, pp. 95-116 (2000).

5. F. Jacob. La Logique du vivant, une histoire de l'hérédité (Gallimard, Paris, 1970).

6. A. Pichot. Expliquer la vie. De l'âme à la molécule (Quae Versailles, 2011).

7. C-T. Wolfe, L'organisme : un concept hybride et polémique. In J-J. Kupiec, La vie et alors? (p. 267 - 281) (Belin, Paris, 2013).

8. J-C. Ameisen, La mort et la sculpture du vivant. In L. Cherlonneix (Ed.), Nouvelles représentations de la vie en biologie et philosophie du vivant. La sculpture du vivant à l'échelle de l'interdisciplinarité (pp.3 - 41) (De Boeck, Bruxelles, 2013).

9. M. Morange, Peut-on définir la vie ? In J-J. Kupiec, La vie et alors ? (p. 41 - 49) (Belin, Paris, 2013).

10. J-N. Tournier, Le vivant décodé : quelle nouvelle définition donner à la vie ? (EDP sciences, Les Ulis, 2005).

11. G. Canguilhem, Article «vie». In Encyclopédia universalis T. 23, (pp. 764 -769). (Vrin, Paris, 1990).

12. J-F. Chevrier, C. Maurice, L'animalité comme envers de l'humain. In F. Burgat (Ed.), L'animal dans nos sociétés (p. 13 - 15). Paris : (Dossier la documentation française, problèmes politiques et sociaux, ${ }^{\circ} 896$, Paris, 2004).

13. E. Baratay, Et l'homme créa l'animal (Odile Jacob, Paris, 2003).

14. J-J. Rousseau, Discours sur l'origine et les fondements de l'inégalité parmi les hommes (Gallimard, Paris, 1990) (1ère éd. 1755).

15. L. Ferry, Le nouvel Ordre écologique, l'arbre, l'animal et l'homme (Grasset, Paris, 1992).

16. C. Larrère, La thèse utilitariste : prendre en compte les intérêts des animaux. In Burgat, F. (Ed.) L'animal dans nos sociétés (p. 38 - 40). (Dossier la documentation française, problèmes politiques et sociaux, $\mathrm{n}^{\circ} 896$, Paris, 2004).

17. J-Y. Goffy, L'animal comme membre de la communauté morale : l'utilitarisme de Peter Singer. In M.-H. Parizeau et G. Chapouthier (Ed). L'être humain, l'animal et la technique (p. 21 44). (Les presses de l'Université Laval, Québec, 2007).

18. P. Singer, L'égalité animale expliquée aux humain-es. Edition numérique tahin-party. http://tahin-party.org/textes/singer.pdf

Première édition française sous le titre : Le Mouvement de libération animale. Sa philosophie, ses réalisations, son avenir éd. Françoise Blanchon, Lyon, (1991)

19. M-A. Hermitte, Le vivant et le droit. Le courrier du CNRS, 75, $41-42$ (1990). 
20. D. Lecourt, Humain, post humain (PUF, Paris, 2003).

21. C. Darwin, L'expression des émotions chez l'homme et les animaux (Complexe, Bruxelles, 1981) (1ère éd. 1872).

22. D. Lestel, L'animal singulier (Seuil, Paris, 2004).

23. P. Vandeginste, Le propre de l'homme et la culture chimpanzée. Le Monde des débats, 26, juin, $22-23$ (2001).

24. F. de Waal, De la réconciliation chez les primates (Flammarion, Paris, 1992).

25. F. de Waal, La politique du chimpanzé (Éditions du Rocher, Paris; Ré édition en 1995 chez Odile Jacob, 1992b).

26. F. de Waal, Le bon singe (Bayard, Paris, 1997)

27. M. Merleau-Ponty, La nature In B. Cyrulnik (Ed.) Si les lions pouvaient parler : essais sur la condition animale (p. 707 - 709) (Gallimard, Paris, 1998).

28. G. Lecointre, H. Le Guyader, Classification phylogénétique du vivant, T.I (Belin, Paris, 2001).

29. M. Majerus, Le papillon de la discorde. Les dossiers de La Recherche, T 02751, 12 - 15. (2007). Et J-M. Lange, In M. Coquidé et S. Tirard (Eds). L'évolution (Vuibert - Adapt, Paris, 2009).

30. Y. Kuster, J-M. Lange, Étude de quelques conceptions autour des mécanismes de l'évolution d'une profession de santé: l'exemple de candidats à un concours de kinésithérapie. In M. Coquidé et S. Tirard (Eds). L'évolution (Vuibert - Adapt, Paris, 2009).

31. M. Morange, Les origines de la vie. Le point de vue d'un historien de la biologie. In M-c. Maurel, \& P-A. Miquel (Eds). Nouveaux débats sur le vivant (p. 23-39) (Kimé, Paris, 2003).

32. S-J. Gould, Interrogé par Marcel Blanc, «Un accident dans l'histoire des espèces », Le Monde des débats, 26, juin (2001).

33. S-J. Gould, Darwin et les grandes énigmes de la vie (Seuil, Paris, 1997).

34. C. Simard, L. Harvey et G. Samson, Épistémologie de la biologie et conceptualisation du vivant chez des futurs enseignants et biologistes, in La "vie » et le "vivant » : de nouveaux défis à relever dans l'éducation, SHS web of conferences 2015 (cet ouvrage).

35. T. Kuhn, La structure des révolutions scientifiques. Paris: Flammarion. (1983).

The structure of scientific revolutions. University of Chicago Press, (1962).

36. H. Atlan, Les Étincelles de hasard, tome 2 : Athéisme de l'écriture (Seuil, Paris, 2003).

37. H. Atlan, La fin du tout génétique? Nouveaux paradigmes en biologie (INRA Éditions, Paris, 1999).

38. H. Kitano, Foundations of Systems Biology (MIT Press, Cambridge, USA, 2001).

39. E. T. Liu, Systems biology, integrative biology, predictive biology. Cell, 121(4): 505- 506 (2005).

40. M. Coquidé, M. dell'Angelo, S. Dorey, C. Fortin, M. Gallezot, S. Henocq, F. Kalali, J-M. Lange, G. Rumelhard, Espace et temps dans les sciences du vivant : de nouvelles perspectives pour la recherche en didactique des Sciences et des Technologies, RDST, 4, 139 - 160 (2012).

41. T. Heams, Existe-t-il un programme génétique ? In J-J. Kupiec, La vie et alors ? (p. 131-146) (Belin, Paris, 2013).

42. http://www.admiroutes.asso.fr/gilbertchauvet/pourquoi.htm

43. J.J. Kupiec, P. Sonigo, Ni Dieu ni gène. Pour une autre théorie de l'hérédité (Seuil, Paris, 2000).

44. J-C. Dupont, L'embryologie : histoire et perspectives. In J-J. Kupiec, La vie et alors ? (p. 131-146) (Belin, Paris, 2013).

45. C. Fortin, «La diversification des êtres vivants sans modification du génome» dans le programme français de la classe de Terminale scientifique: enjeux didactiques et épistémologiques, dans La "vie » et le "vivant» : de nouveaux défis à relever dans l'éducation, SHS web of conferences 2015 (cet ouvrage)

46. J.J. Kupiec, Une approche darwinienne de l'ontogenèse. Les mondes darwiniens (Syllepse, Paris, 2009). 
La « vie » et le « vivant » : de nouveaux défis à relever dans l'éducation

47. J.J. Kupiec, L'identification cellulaire. In J.J. Kupiec, et P. Sonigo (Eds). Ni Dieu ni gène. Pour une autre théorie de l'hérédité (p. 85-128) (Seuil, Paris, 2000).

48. A-M. Moulin, Histoire croisée de la microbiologie et de l'immunologie. In J-J. Kupiec, La vie et alors? Débats passionnés d'hier et d'aujourd'hui (p. 147-176) (Belin, Paris, 2013).

49. T. Pradeu, Critique du soi et du non-soi en immunologie. In J-J. Kupiec, La vie et alors ? Débats passionnés d'hier et d'aujourd'hui (p. 177 - 190) (Belin, Paris, 2013).

50. M. Benasayag, P-H. Gouyon, Fabriquer le vivant (La Découverte, Paris, 2012).

51. G. Rumelhard, Groupe d'étude, "Évolutions des sciences de la vie et de la santé et enjeux de formation », UMR STEF ENS de Cachan-INRP (22 mai 2015). 
